\title{
Unilateral Bone Window Cerebral Falx Incision of Bilateral Frontal Lobes Cerebral Contusion and Laceration under Neuroendoscopy and Literature Review
}

\author{
Gang Yang, Shaojun Yang, Chao Gu, Chenbing Wang, Lulu Weng \\ Department of Neurosurgery, Zhuji Affiliated Hospital of Shaoxing University, Shaoxing, China \\ Email: tekeyang@sina.com
}

How to cite this paper: Yang, G., Yang, S.J., Gu, C., Wang, C.B. and Weng, L.L. (2021) Unilateral Bone Window Cerebral Falx Incision of Bilateral Frontal Lobes Cerebral Contusion and Laceration under Neuroendoscopy and Literature Review. Open Journal of Modern Neurosurgery, 11, 164-170.

https://doi.org/10.4236/ojmn.2021.113020

Received: May 26, 2021

Accepted: July 12, 2021

Published: July 15, 2021

Copyright $\odot 2021$ by author(s) and Scientific Research Publishing Inc. This work is licensed under the Creative Commons Attribution International License (CC BY 4.0).

http://creativecommons.org/licenses/by/4.0/

\begin{abstract}
Background: Bilateral frontal lobes cerebral contusion and laceration is one unique brain injury in neurosurgery department. It is characteristic of recessive attacking and develops quickly. The unilateral cerebral falx incision is a new minimally invasive surgery that can solve bilateral frontal lobes cerebral contusion and laceration in one surgery. However, it has some limitations in removal of contralateral frontal hematoma and hemostasis due to the limited field of view under the microscope. The unilateral bone window cerebral falx incision of bilateral frontal lobes cerebral contusion and laceration under a neuroendoscopy can acquire a good illumination and field of view. This is beneficial to complete removal of contralateral hematoma, effective hemostasis and retaining brain tissue functions to the maximum extent. Case Presentation: The patient, a 55-year-old man, was hospitalized for "consciousness disorder by $12 \mathrm{~h}$ because of car accident". Physical Examination: Coma, GCS score of E1V2M5, bilateral pupil diameter of $2 \mathrm{~mm}$, presence of light response, contusion of scalp at the left top, peripheral dysphoria and bilateral Bartter syndrome negative. The patient has a history of non-traumatic cerebral stroke 3 years ago. Head CT: Longitudinal fracture of frontal parietal occipital bone, bilateral frontal lobes contusion and laceration, subarachnoid hemorrhage. Diagnosis: Bilateral frontal lobes contusion and laceration, longitudinal fracture of frontal parietal occipital bone, subarachnoid hemorrhage and hematoma of scalp. In emergency treatment, unilateral bone window cerebral falx incision of bilateral frontal lobes cerebral contusion and laceration under a neuroendoscopy was performed. The surgery has achieved satisfying effect. Discussion: This case realized the goal of removing contralateral frontal hematoma through unilateral craniotomy under a neuroendoscopy. Due
\end{abstract}


to the clear field of view, it retained extracerebral layer structures of contralateral olfactory nerve protection frontotemporal completely. Moreover, this surgical technique is conducive to intraoperative recognition of pericallosal arteries and lateral fractured blood vessels. It also involves protection, which conforms to the minimally invasive philosophy. The proposed surgical technology can eliminate contralateral frontal hematoma under a good field of view. However, it is suggested not to manage with the further operation on patients who have brain swelling and difficulties in exposure of cerebral falx. These patients need to determine causes of brain swelling and choose bilateral craniectomy if necessary. Conclusions: Unilateral bone window cerebral falx incision of bilateral frontal lobes cerebral contusion and laceration under a neuroendoscopy is a new application of minimally invasive philosophy in craniocerebral injury operation. It still needs further clinical verifications and experience accumulation.

\section{Keywords}

Neuroendoscopy, Cerebral Falx Incision, Bilateral Frontal Lobes Cerebral Contusion and Laceration

\section{Background}

Bilateral frontal lobes cerebral contusion and laceration is one unique brain injury in neurosurgery department and it is mainly caused by the decelerating contrecoup injury of posterior occipital force. It is characteristic of recessive attacking and develops quickly. It has been a high concern of physicians [1]. In particular, given an asymmetric bilateral frontal lobes cerebral contusion and laceration, the side of mild injury is easy to have delayed intracranial hematoma due to influences by decompression at the operation side. Hence, bilateral frontotemporal flap craniotomy is needed [2]. Nevertheless, a large cranial defect is left at the frontal part after the bilateral frontotemporal flap craniotomy. This causes a large trauma and adverse impacts on prognosis, and influences beauty of patients [3]. Clinical treatment for bilateral frontal lobes cerebral contusion and laceration also tends to minimally invasive. Unilateral cerebral falx incision, a new minimally invasive operation, can solve bilateral frontal lobes cerebral contusion and laceration in one surgery. It has attracted high clinical attention. Patients can shorten operation time, decrease intraoperative blood loss volume, accelerate postoperative rehabilitation and improve prognosis significantly after unilateral cerebral falx incision [4]-[10]. However, it has some limitations in removal of contralateral frontal hematoma and hemostasis due to the limited field of view under the microscope. With promotion and development of neuroendoscopy technology, the unilateral bone window cerebral falx incision of bilateral frontal lobes cerebral contusion and laceration under a neuroendoscopy can acquire a good illumination and field of view. This is beneficial to complete 
removal of contralateral hematoma, effective hemostasis and retaining brain tissue functions to the maximum extent.

\section{Case Presentation}

Case Presentation: the patient, a 55-year-old man, was hospitalized for "consciousness disorder by $12 \mathrm{~h}$ because of car accident". Physical examination: coma, GCS (Glasgow Coma Scale) score of E1V2M5, bilateral pupil diameter of 2 $\mathrm{mm}$, presence of light response, contusion of scalp at the left top, peripheral dysphoria and bilateral Bartter syndrome negative. The patient has a history of non-traumatic cerebral stroke 3 years ago. Head CT (Computed Tomography): longitudinal fracture of frontal parietal occipital bone, bilateral frontal lobes contusion and laceration, subarachnoid hemorrhage (Figure 1). A brain reexamination was performed at $6 \mathrm{~h}$ after the hospitalization (Figure 2). CT Diagnosis: bilateral frontal lobes contusion and laceration, longitudinal fracture of frontal parietal occipital bone, subarachnoid hemorrhage and hematoma of scalp.

According to the head CT performances at $6 \mathrm{~h}$ after the hospitalization, bilateral frontal lobes contusion and laceration were worsened. The patient was still in a coma and GCS score was E1V2M5. In emergency treatment, unilateral bone window cerebral falx incision of bilateral frontal lobes cerebral contusion and laceration under a neuroendoscopy was performed. The patient took the supine position with the head inclined toward the left. The side with large hematoma was used as the modified pterio-approach and the one hole was drilled near the midline forehead zygomatic process and supratemporal line, respectively. One hole was close to the anterior cranial fossa and the frontal sinus was not opened. The incision was made across the midline of the forehead to expose the anterior cranial fossa fully. Intracranial hematoma and cerebral contusion and laceration at one side of frontal pole were eliminated under a microscope. The frontal pole had a space and the cerebral falx and anterior cranial fossa were exposed. After the contralateral hematoma and cerebral contusion and laceration at the skull base were eliminated along the hole under the falx, the cerebral falx was cut longitudinal along the cockscomb to about $3 \mathrm{~cm}$ of the inferior wall of superior sagittal sinus. Pay attention to protect superior sagittal sinus and cerebral falx bleeding points were treated by electrocoagulation. Contralateral frontal hematoma and inactivated brain tissues were eliminated under the good direct field of view of a neuroendoscopy, while the contralateral olfactory nerves were retained. The anterior cerebral arteries and vessel branches were protected and hemostasis was performed properly. Next, the dura mater rupture at skull base was closed by medical adhesive bandage to prevent leakage of cerebrospinal fluid. The cerebral falx was not stitched up. The dura mater was repaired by artificial dura mater and bone flap was removed for decompression. Conventional treatment was implemented after the operation. Head CT was performed again at $16 \mathrm{~h}$ after the operation, finding satisfying operation effect (Figure 3). 

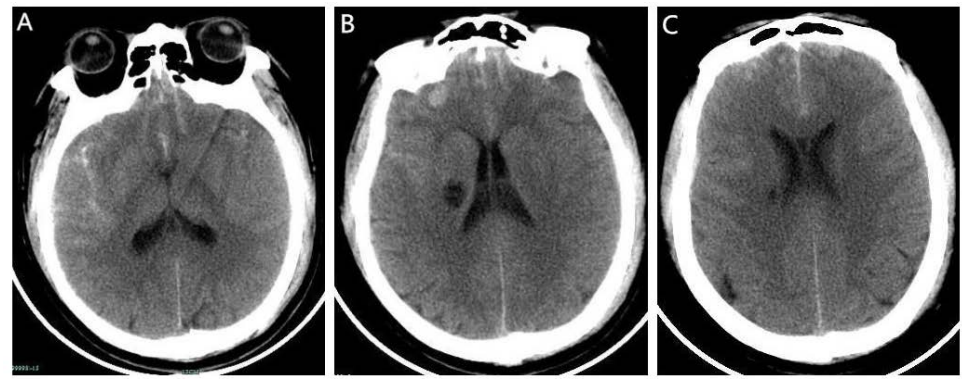

Figure 1. Head CT performance at hospitalization: bilateral frontal lobes contusion and laceration and subarachnoid hemorrhage.
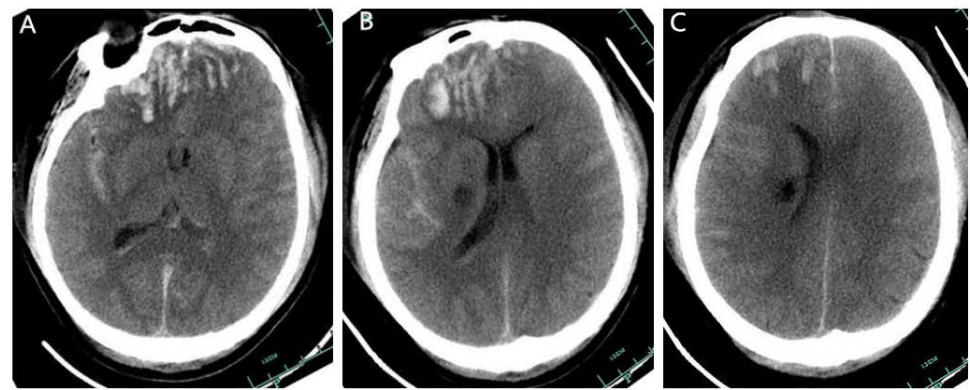

Figure 2. Head CT performance at $6 \mathrm{~h}$ after hospitalization: bilateral frontal lobes contusion and laceration worsening.
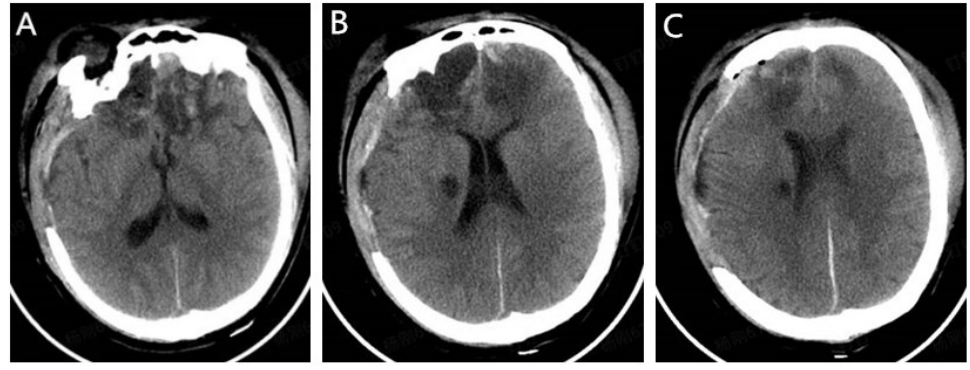

Figure 3. Head CT performance after the operation: bilateral frontal lobes contusion and laceration removed and intracranial pressure dropped significantly.

\section{Discussion}

Bilateral frontal lobes cerebral contusion and laceration is a unique type of neurosurgical critical disease. Due to the unique injury site, patients have mild consciousness disorder in early stage and insignificant symptoms. If no enough clinical attention is provided, it is easy to cause diffusive cerebral swelling with the progress of the disease. Moreover, the disease will exacerbate suddenly and even cause cerebral hernia or breath arrest, thus losing the best time for operation. Recently, some studies have reported that bilateral frontal lobes cerebral contusion and laceration is a relatively serious type of closed brain injury and most cases are caused by decelerating contrecoup injury of posterior occipital force. It is often seen in car accidents and fall injuries. Most patients have severe and complicated injury conditions. On one hand, the broken brain tissues and intracerebral hematoma form a space-occupying lesion. On the other hand, 
there's cerebral edema. Therefore, the patients can only be saved by surgery in most cases.

It is relatively difficult to make a decision on clinical surgery for bilateral frontal lobes cerebral contusion and laceration. If patients choose conservative therapy, the disease will exacerbate suddenly and patients lose the best time for operation. If they choose surgery in early stage, the bilateral craniotomy usually causes a large trauma which is difficult to be accepted. Unilateral cerebral falx incision often has insufficient field of view for contralateral hematoma removal under a microscope and it is difficult to perform hemostasis. This also is relatively conservative in contralateral hematoma removal, which is also disadvantageous against protection of contralateral frontal lobe functions. Using the accurate surgical scheme is the key to control the disease, store and recover neurological functions, increase the quality of life and save life of patients. Minimally invasive has become an important direction of neurosurgery development. it has transited from previous emphasis on decreasing death rate to decreasing surgical injury and promoting functional recovery. The minimally invasive philosophy has expanded to all fields of neurosurgery, including surgery for craniocerebral injuries. In this study, the patient realized the goal of contralateral lobe hematoma removal through a unilateral craniotomy under a neuroendoscopy. Due to the clear field of view, he retained extracerebral layer structures of contralateral olfactory nerve protection frontotemporal completely. Moreover, the proposed surgical technology is conducive to intraoperative recognition of pericallosal arteries and lateral fractured blood vessels. It also involves protection, which conforms to the minimally invasive philosophy.

Unilateral bone window cerebral falx incision of bilateral frontal lobes cerebral contusion and laceration under a neuroendoscopy has to assure enough operation space for neuroendoscopy. To address this problem, it often uses modified pterio-approach or frontotemporal approach usually near the forehead midline. In the operation, sphenoid ridge is treated well. After frontopolar hematoma and inactivated brain tissues are eliminated, there's some space in anterior frontal lobe and the frontal pole is easy to be pulled open backward. The skull base and frontal part of cerebral falx can be exposed well by a gentle traction. If there's evident cerebral edema, the lateral fissure cistern of one side can be opened to release cerebrospinal fluid slowly to lower the intracranial pressure. This method can expose the anterior skull base, knee part of the callosum and anterior cerebral falx fully.

Some Chinese scholars have pointed that the proposed surgery shall be used cautiously under following conditions: 1) patients who have contralateral hematoma $>15 \mathrm{ml}$ or far away from the midline, and hematoma close to lateral fissure. This is because exposure to the lateral fissure is the limit of the surgery and it is more difficult to achieve hemostasis near the lateral fissure; 2) patients who still have serious brain swelling after hematoma removal at one side and are difficult to expose the cerebral falx. Patients who still have high intracranial pressure and brain swelling after the operation shall use conventional decompressive 
craniectomy; 3) patients who have obvious hemorrhagic tendency and extensive hemorrhage in early stage of hospitalization. Since the hemorrhage range is often not explicit in early stage and it is also difficult to determine the excision extension in operation, it is easy to have hematoma recurrence after the operation [11]. The author believed that the proposed surgical technology can eliminate contralateral frontal hematoma under a good field of view, but it is not suggested to manage with the operation for patients who have brain swelling and have difficulties in cerebral falx exposure. Instead, it has to recognize causes of brain swelling and bilateral craniotomy has to be performed if necessary. For patients who have obvious hemorrhagic tendency and extensive hemorrhage in early stage of hospitalization, it is reported that most patients have traumatic coagulation. The incidence rate of coagulation in adult patients with cerebral trauma is about $42 \%$ and the death rate of patients who have combined coagulation and cerebral trauma is as high as $45.5 \%$ [12]. The incidence rate of acute traumatic coagulation in patients with severe craniocerebral injury is $61.1 \%$ [13]. During perioperative period, patients have to pay attention to replacement of blood coagulation factors and fibrinogen to correct the coagulation functions. Subsequently, unilateral bone window cerebral falx incision of bilateral frontal lobes cerebral contusion and laceration under a neuroendoscopy is still a good choice.

\section{Conclusion}

The unilateral bone window cerebral falx incision of bilateral frontal lobes cerebral contusion and laceration under a neuroendoscopy is a new application of minimally invasive philosophy in craniocerebral injury. It is worthy of further explorations and studies.

\section{Fund-Supported Projects}

Zhejiang Medical Association Clinical Research Fund Projects 2019ZYC-A157; Shaoxing Health Science and Technology Plan 2017CX025; Zhejiang Health Development Foundation 2019ZD059; Zhejiang Health Development Foundation 2019ZA127.

\section{Conflicts of Interest}

The authors declare no conflicts of interest regarding the publication of this paper.

\section{References}

[1] Yuan, S., Xiao, W., Li, G., et al. (2015) Applications of Continuous Intracranial Pressure Monitoring in Treatment of Bilateral Frontal Lobes Cerebral Contusion and Laceration. Journal of Hainan Medical College, 26, 3535-3536.

[2] Li, B., Huang, Q., Wang, Z., et al. (2013) Treatment of Bilateral Frontal Lobes Cerebral Contusion and Laceration Based on Decompressive Craniotomy by Bilateral Coronary Incision and Intracranial Pressure Monitoring. Journal of Practical Medicine, 29, 4053-4056. 
[3] Fang, X., Li, C., Fu, R, et al. (2009) Clearance of Cerebral Contusion and Laceration at Bilateral Lobes and Intracranial Hematoma through Unilateral Cerebral Falx Incision. Chinese Journal of Microinvasive Neurosurgery, 14, 420-421.

[4] Huang, G., Yu, S., Yi, Y., et al. (2017) Advantages and Disadvantages of Unilateral Cerebral Falx Incision under a Microscope and Bilateral Craniotomy with a Bone Window in Treatment of Bilateral Frontal Lobes Cerebral Contusion and Laceration. Practical Clinical Journal of Integrated Traditional Chinese and Western Medicine, 17, 122-123.

[5] Hong, Y. (2012) Analysis on Therapeutic Effect of Unilateral Cerebral Falx Incision to Remove Bilateral Frontal Lobes Cerebral Contusion and Laceration with Complications of Intracranial Hematoma. Guide of China Medicine, No. 24, 228.

[6] Li, Z. (2020) Effects of Unilateral Cerebral Falx Incision on Postoperative GOS Score and Length of Stay of Patients with Bilateral Frontal Lobes Cerebral Contusion and Laceration. Capital Food and Medicine, 27, 29.

[7] Li, X. and Liu, S. (2011) Unilateral Cerebral Falx Incision Treatment of Bilateral Frontal Lobes Cerebral Contusion and Laceration (Attached a Report of 30 Cases). Chinese Journal of Clinical Neurosurgery, 16, 427-428.

[8] Yu, Y., Deng, Z., Jiang, J., et al. (2019) Comparison of Effects between Unilateral Cerebral Falx Incision and Bilateral Bone Window Craniotomy in Bilateral Frontal Lobes Cerebral Contusion and Laceration. Clinical Medical Engineering, 26, 1671-1672.

[9] Tang, T. and Hu, G. (2016) Comparison of Clinical Therapeutic Effects of Different Surgical Technologies to Bilateral Frontal Lobes Cerebral Contusion and Laceration. Journal of Hainan Medical College, 27, 3035-3037.

[10] Li, G., Yuan, S., Luo, W., et al. (2013) Clinical Study on Unilateral Cerebral Falx Incision under a Microscope in Treatment of Bilateral Frontal Lobes Cerebral Contusion and Laceration. Chinese Disability Medicine, No. 8, 145-146.

[11] Xu, X., Wei, X., Chen, Z., et al. (2012) Therapeutic Effect of Unilateral Bone Window Cerebral Falx Incision in Asymmetric Bilateral Frontal Lobes Cerebral Contusion and Laceration and Its Influences on Frontal Lobe Functions. Chinese Journal of Trauma, 28, 162-164.

[12] Dekker, S.E., Duvekot, A., de Vries, H.M., et al. (2016) Relationship between Tissue Perfusion and Coagulopathy in Traumatic Brain Injury. Journal of Surgical Research, 205, 147-154. https://doi.org/10.1016/j.jss.2016.06.023

[13] Chhabra, G., Sharma, S., Subramanian, A., et al. (2013) Coagulopathy as Prognostic Marker in Acute Traumatic Brain Injury. Journal of Emergencies, Trauma, and Shock, 6, 180-185. https://doi.org/10.4103/0974-2700.115332

\author{
Abbreviations \\ GCS: Glasgow Coma Scale \\ CT: Computed Tomography
}

